\title{
¿Es la gastronomía un arte?
}

\author{
Is gastronomy an art?
}

TEÓFILO HAMUI WAYSEL ${ }^{1}$

(10) https://orcid.org/0000-0002-3351-1865

Artículo de investigación

Recepción: 13 de enero de 2020

Aceptación: 03 de septiembre de 2020

Cómo citar este artículo

Hamui Waysel, T. (2020). ¿Es la gastronomía un arte? Sosquua. Revista Especializada en Gastronomía 2(1), pp. 09-27 Recuperado a partir de: http://cipres.sanmateo.edu.co/

index.php/sosquua

Reconocimiento-SinObraDerivada 4.0 Internacional (CC BY-NC-ND)

1Universidad Iberoamericana. Correo electrónico: thamui@hotmail.com 


\title{
Resumen:
}

La comida ha ocupado un lugar central en cualquier actividad humana, desde las formas de organización más primarias, hasta las actividades políticas y económicas más complejas. Ocupa un lugar central en cualquier proceso histórico; no solo se trata de la satisfacción de una necesidad fisiológica, también una forma de expresión; implica la técnica de un "genio" y una experiencia estética por parte del comensal o espectador. Así, el presente artículo reflexiona sobre la posibilidad de considerar a la gastronomía como un arte. Si es así, ¿por qué los teóricos del arte nunca la han considerado como tal? En este sentido, se analiza el rol que ha jugado la alimentación en la configuración de la política, la medicina y hasta la cultura misma. Por otra parte, menciona algunos de los criterios empleados para determinar qué es el arte en los distintos momentos históricos. Por último, se revisan aquellos elementos determinantes de la gastronomía que pudieran definir si es (o no) un arte.

Palabras clave: arte; gastronomía; técnica; estética; ciencia.

\begin{abstract}
:
Food has always occupied a central place in any human activity, from the most primary organization forms, to the most complex political and economic activities. It occupies a central place in any historical process; it is not just about the satisfaction of a physiological and biological necessity, but of a form of expression; always involving the technique of a genius and an aesthetic experience on the part of the spectator. Thus, this article reflects about the possibility of consider the gastronomy as an art. If so, why art theorists have never considered it in that way? In this sense, it makes an analysis regarding the role that food has played in the configuration of both politics, medicine and even culture itself. In the other hand, the article mentions some of the criteria that, in the different historical moments they have used to determine what is art. Finally, we carry out a review of determining elements of gastronomy that could define whether it is an art.
\end{abstract}

Keyword: art; gastronomy; technique; esthetic; science. 


\section{Introducción}

Cualquier actividad humana, sea cual fuere su naturaleza, desde las primeras formas de organización, hasta los planteamientos económicos, científicos o políticos actuales, han encontrado siempre a la comida como uno de sus elementos y motivaciones más medulares.

En perspectiva, al echar un simple vistazo al desarrollo del ser humano, así como a cualquier proceso histórico en el que esté involucrado, se puede advertir el carácter central que ocupa la alimentación en cualquier tipo de organización o desarrollo del humano. Lo anterior, ya sea que se analice desde el punto de vista evolutivo, biológico, moral, o cualquier otro; el ser humano simplemente no existe sin alimento.

Desde las organizaciones políticas más primitivas, inclusive previas a los asentamientos humanos y a la agricultura, las relaciones de los seres humanos han girado en torno a la alimentación. Desde aproximadamente dos (2) millones de años, cuando los primeros homínidos comenzaron a desarrollarse; posteriormente, hace aproximadamente 70 mil años, cuando los homo sapiens desarrollaron cierto lenguaje primitivo; las formas de organización de los grupos errantes de humanos eran basadas siempre en la búsqueda de comida. Entonces, los roles que adoptaron con respecto a la caza y a la recolección, hasta su desplazamiento por las llanuras, los bosques (incluso entre un continente y otro), siempre fue buscando satisfacer su necesidad evolutiva de la alimentación.

Bajo este marco, existen hipótesis tendientes a demostrar que el desarrollo de los primeros utensilios fue motivado por la necesidad de alimentarse. Además, se piensa que uno de los usos más comunes de los primeros utensilios de piedra fue romper huesos con el fin de llegar a la médula. De la misma forma que el pájaro carpintero usa su pico para extraer insectos de los troncos, los primeros homo sapiens se alimentaban del tuétano. Esta fue la razón por la cual se vieron en la necesidad de desarrollar herramientas, es decir, por su precariedad física.

Entonces, si se parte de la suposición según la cual los homo sapiens se encuentran a una manada de leones devorando un animal. Tras esperar pacientemente a que los leones terminen de comer, tienen que esperar que las hienas terminen, para posteriormente esperar a los buitres y solo entonces era su turno (Harari, 2014). Así, resulta sumamente interesante la organización política 
primigenia de los humanos; además, incluso el desarrollo de la técnica nace a partir de la necesidad humana por conseguir alimento.

De esa manera, resulta evidente la forma en que los cambios en la organización de los seres humanos han girado siempre en torno a la alimentación. No solo ello, sino que una vez se complejizaron estas formas de organización y conseguir alimentos, fue esta necesidad la que dio nacimiento a la cultura misma.

Lo anterior es así pues se debe tener siempre presente que el nacimiento de todas estas formas de relacionarse (no solo con otros individuos, sino con cualquier otro objeto que se encuentre a nuestro alrededor) no es más que el instinto de supervivencia del ser humano. Es decir, al encontrarse en una posición de debilidad con respecto al entorno propio, no queda más alternativa que encontrar los medios para sobrevivir, sean naturales o no.

Esto no quiere decir que la cultura naciera como una especie de mecanismo evolutivo para la supervivencia, o que cualquier otro animal que se encuentre en una situación tan precaria como la nuestra pueda desarrollar una estructura como lo que entendemos como cultura. En cambio, se encontró en la posibilidad de hallar la forma de preservar su existencia derivado de las capacidades cognitivas de las cuales el homo sapiens fue dotado por los procesos evolutivos que tuvieron lugar.

\section{La gastronomía y la cultura}

Desde su nacimiento, el ser humano siempre ha sido más lento, más débil y menos capaz de defenderse a sí mismo. De no haber desarrollado cierta manera de relacionarse con su medio ambiente, inevitablemente habría corrido la suerte de, por un lado, ser la presa más fácil para los depredadores. Por otro lado, de las lluvias, las altas y las bajas temperaturas, así como cualquier otra dificultad que su mismo entorno pudiera presentar, es decir, la extinción. En este sentido, la cultura nace como la forma más violenta de negar a la naturaleza o, lo que es más, de dominarla y hasta de domesticarla cuando exista la posibilidad de hacerlo.

Derivado de lo anterior, el antropólogo francés Claude Lévi-Strauss (2012) sostiene que la cultura nace precisamente en el momento en que, derivado de los procesos y cambios cognitivos que tuvieron lugar con el humano, se dio cuenta de 
la posibilidad de cocinar la comida y de la diferencia que existe entre la comida cruda y la comida cocida (Lévi-Strauss, 2012). Incluso describe algunos mitos en los cuales se hace especial énfasis en la importancia de la digestión y el tratamiento a la carne de los animales. Esto evidencia cómo ciertas ritualidades y mitos fundacionales tienen como epicentro a la comida.

Por otra parte, vale la pena mencionar la forma tan radical en la que cambia la comprensión del ser humano de su ambiente desde el momento de la domesticación del fuego. Como señala Lévi-Strauss, pareciera que lo cocido es la transformación cultural de lo crudo, mientras que lo podrido es su transformación natural.

En efecto, tanto Lévi-Strauss como otros autores señalan que la cultura nació justamente cuando se inició la domesticación del fuego. A partir de allí, los humanos comenzaron no solo a contar con una fuente de luz y de calor, sino que revolucionó por completo la manera de relacionarse con la naturaleza. En particular, por primera vez pudieron explotarla y alimentarse de ella libremente y casi sin ningún riesgo.

Lo anterior, pues se especula que incluso los primeros humanos pudieron llevar a cabo deliberadamente incendios controlados; les permitía convertir espesuras intransitables e improductivas en praderas relativamente amplias con abundante caza. Allí podrían caminar una vez que el fuego se hubiera extinguido, así como recolectar animales, nueces y tubérculos quemados (Harari, 2014).

Sumado a esto, ciertos alimentos que el ser humano no podía digerir crudos (como el arroz o el trigo) ahora se encontraban en la posibilidad ser masticados y digeridos con mayor facilidad. No era necesario invertir tanto tiempo en las actividades alimenticias, por ejemplo, mientras un chimpancé dedica cinco (5) horas diarias en promedio para masticar. Un homo sapiens podría destinar una sola hora y ocupar más tiempo en el desarrollo de otras actividades (Harari, 2014). Sin duda, esto tuvo una importante injerencia en el desarrollo de la técnica, del lenguaje y de la cultura misma; además estos alimentos se convirtieron en elementos esenciales de nuestra dieta.

Por consiguiente, resulta difícil encontrar un ejemplo más claro para entender a la cultura como la forma de negación y dominación de la naturaleza empleada por el ser humano para su propia supervivencia. Es decir, humanos quemando bosques deliberadamente y, como se ha evidenciado, dicha actividad fue siempre llevada a cabo con el fin de conseguir alimentos. 


\section{La alimentación y la historia}

Ahora bien, desde la prehistoria, en la más remota antigüedad y, hasta nuestras fechas, la alimentación ha sido parte esencial todas las formas de relacionarnos. Sin embargo, resulta sumamente interesante preguntarse cuál es la forma de conocimiento mediante la cual entenderla mejor. Es decir, vale la pena preguntarse si la mejor manera de entender a la dietética es por medio de la ciencia, el arte, la política, o simplemente entenderla como la necesidad de saciar nuestra necesidad biológica y evolutiva de alimentarnos.

No cabe duda de que al momento de preparar cualquier tipo de alimento, ya sea crudo, cocido, asado o incluso madurado o podrido, se deben de emplear conocimientos científicos. Una vez cambia su estado inicial, inevitablemente se emplean las leyes de la física y, al modificarlo mediante la utilización de calor, se utiliza la química. Empleando desde las formas más básicas de las ciencias hasta las más complejas.

Adicionalmente, no podemos perder de vista la estrecha relación que ha existido históricamente entre los regímenes alimenticios y la ciencia, por ejemplo, en la antigüedad no existió nunca una separación clara entre la dietética y la medicina. Como se evidencia, algunos de los más celebres médicos de la antigüedad basaban gran parte de sus estudios científicos en el régimen dietético para cada persona. Asimismo, según las características y malestares de cada individuo determinaban qué nuevo régimen alimenticio debería de adoptar.

Un claro ejemplo de ello fue el distinguido médico Hipócrates. En su obra Sobre la dieta se propone llevar a cabo un análisis que le permita teorizar sobre las actividades que el ser humano debe llevar a cabo para conservar su salud. Analiza tanto el tipo de alimentos que deben ser ingeridos, como la actividad física que debe ser realizada. Así, para Hipócrates existen dos (2) elementos primordiales en la configuración física del ser humano: el agua y el fuego. Explica que el agua es necesaria para nutrirse y el fuego para moverse, por lo que necesitamos un equilibrio entre estos para contar con buena salud.

El médico griego explica que la humanidad debe nutrirse para poderse mover, pero si se nutre en exceso no sería posible moverse. Lo mismo sucedería si existe una actividad física en exceso sin una nutrición adecuada. En consecuencia, se debe hallar una media exacta entre la cantidad de actividad física a realizar en relación con la nutrición para llevar una vida adecuada (Hipócrates, Sobre la dieta, Libro I). 
En ese sentido, al dar un simple vistazo a cualquier tratado médico de la antigüedad (incluso a una prescripción médica de la edad media) se puede interpretar que la dietética era entendida como medicina. De hecho, se pensaba que determinadas enfermedades eran causadas por la ingestión en exceso de algunos alimentos; asimismo, se veía a la indigestión como uno de los más terribles males que pudiera sufrir el cuerpo.

\section{La alimentación y la nutrición}

No solo ello, al pensar en dietética también era necesario pensar en regímenes de ejercicio y hasta de sexualidad, es decir, no se pensaba a la alimentación como un factor aislado; únicamente como una parte del régimen de salud que cualquier persona debería adoptar. Sin embargo, no fue hasta la modernidad en que fue llevada a cabo una escisión clara entre la ciencia en general y la medicina. Lo anterior indica que la alimentación ha sido pensada (por más tiempo), únicamente, como una parte más del estudio de la ciencia y no como un estudio en sí misma.

Lo anterior, sin tomar en cuenta el auge que han tenido los estudios sobre nutrición en las últimas décadas. Desde el siglo XX ha existido una obsesión bien conocida, relacionada con la contabilización de las calorías ingeridas por una persona y la forma en que los procesos químicos tienen lugar en el cuerpo. Según lo expuesto, el estudio de la dietética se ha abordado en diferentes momentos históricos, desde la ciencia, la política, la filosofía y muchas otras formas de conocimiento.

Sin embargo, no hay que perder de vista la importancia que siempre ha tenido la alimentación en cuanto a su carácter estético. Como ya se ha repasado, desde la primera antigüedad los seres humanos se preocuparon por lo que comían y por la calidad de sus alimentos. Asimismo, incluso antes de la domesticación del fuego, debió de existir cierta predilección subjetiva entre un alimento y otro. En ese sentido, se puede entender a la gastronomía como la rama de la dietética ocupada de entender su carácter estético.

Esto nos hace preguntarnos en primer lugar, si podemos considerar estos procesos de cocción y recolección de alimentos como algún tipo de gastronomía primera; es decir, si es que fue por medio de estos procesos, que se empezó a pensar por primera vez en el carácter estético de la alimentación, y si es así, qué tipos de juicios estéticos eran efectuados. 
Adicionalmente, no puede quedar duda de que en caso de que varios alimentos fueran recogidos, lo cual era precisamente el objetivo de estos incendios, los alimentos eran repartidos o escogidos en alguna forma determinada, lo que sugiere incluso que la esteticidad de los alimentos, precede al carácter político de la dietética, pues al determinar quién debe comer primero, o quien debe comer cada cosa, suponemos alguna forma de organización jerárquica o al menos una organización que indicara qué lugar ocupa cada miembro de este tipo de sociedades para que esta pueda funcionar.

Además, desde la prehistoria fue empleada la técnica de buscar los mejores alimentos posibles; ya sea mediante la elaboración de herramientas para extraerlos de algún hueso o algún refugio. Asimismo, mediante la utilización del calor para cambiar las propiedades físicas del alimento a fin de que este pudiera ser ingerido y digerido con mayor facilidad. Un ejemplo claro de los juicios estéticos empleados al pensar en alimentación desde la prehistoria, hasta la fecha, es la forma en que la mayoría de las sociedades prohíben o ignoran distintos animales, plantas o insectos, que en otras partes son consideradas como importantes fuentes de alimentos.

Adicionalmente, cualquier potencial alimento, no solo por encontrarse en abundancia en algún lugar determinado, es utilizado por los habitantes de ese lugar. Es decir, en Francia resulta difícil consumir los insectos o sus larvas, mientras en ciertas sociedades de América, Asia o África son muy apreciados como alimentos. O bien, lo que actualmente pude ser considerado como algo no apetecible gastronómicamente pudo haber sido muy codiciado en algún otro momento histórico. De vuelta a Francia, como los campesinos de borgoña apreciaban la carne de zorro en el siglo XVIII; asimismo, las ratas, los ratones y otros roedores eran consumidos y codiciados durante el sitio de Paris de 1870 (Flandrin, 1987).

\section{La estética en la alimentación}

El anterior apartado evidencia cómo la alimentación ha sido dotada de un carácter estético. Aunque no existan muchas evidencias que sugieran con mucho detalle cuáles eran los alimentos preferidos por los primeros cazadores-recolectores (incluso de la nobleza en la alta edad media) ha existido una preferencia por un tipo de alimento u otro. Por otra parte, también existe quien remonta el estudio y evolución de la primera gastronomía a los griegos y los egipcios. La nobleza y la 
aristocracia designaba como cocineros a los que más conocían sobre los alimentos. No obstante, vale la pena preguntarse si se trató únicamente de una técnica científica o si verdaderamente se trataba del genio del cocinero.

Al respecto, el francés J.A. Brillar-Savarin, quien perteneció a las clases más burguesas a lo largo de su vida. Por lo tanto, gozó siempre de los más grandes lujos gastronómicos. Así, se dio a la tarea de teorizar algunos aspectos importantes de la gastronomía. Además, desde su definición de gastronomía brindó hasta sus dimensiones políticas y económicas. En su obra, el francés expresa su gusto por el placer de comer y describe su goce al hablar de los alimentos y de su preparación; tanto así que cualquier lector queda enamorado de la cocina y de la experiencia culinaria.

Sin embargo, la estética que existe en los alimentos nunca deja de tratar a la gastronomía como una ciencia; incluso señala la necesidad de emplear ciencias como la química y la física para la cocina. Estas deben utilizarse para determinar las propiedades de los elementos con los que se pretenda preparar algún platillo para determinar la forma de manipulación. Sostiene que la última etapa en el conocimiento de la gastronomía es identificar para qué y con qué fin puede ser utilizado cada alimento, a lo que él denomina como la "farmacia" (Savarin, 2016).

Lo anterior resulta sumamente interesante, tanto así que uno de los grandes teóricos de los placeres que representa la alimentación concibe a la gastronomía como una ciencia. Asimismo, hace preguntarse el por qué, al tratarse de una experiencia tan estética en la que participan todos nuestros sentidos, en ningún momento es considerada un arte.

\section{La gastronomía y el arte}

Es cierto que para la elaboración de cualquier platillo son involucrados distintos procesos científicos, y quizá mediante la teorización de estos procesos se podría plantear una técnica que permita llevar a cabo una estandarización en la preparación de los alimentos que cualquier persona pudiera emplear. Considerar estos factores como decisivos para sostener que la forma idónea de entender a la gastronomía sería como a una ciencia, resultaría lo mismo que pensar en la pintura, la escultura o incluso la música como ciencias. Quién podría negar que mediante el 
uso de la geometría cualquier persona podría llevar a cabo el retrato más exacto o que por medio de la utilización de la química se podría generar la combinación de colores para que un paisaje pareciera absolutamente real.

Esto sería como decir que las ilustraciones del cuerpo humano, llevadas a cabo el mismo Da Vinci, resultan únicamente parte del estudio biológico y fisiológico que el florentino llevó a cabo. Entonces, ipor qué nunca ha sido la gastronomía considerada como un arte, si siempre ha sido uno de los saberes que más busca ser experimentado por los sentidos? Para brindar una respuesta a este cuestionamiento es necesario señalar, en primer lugar, qué se entiende por arte. De lo contrario resultaría imposible determinar si la gastronomía puede o no ser considerada como tal.

Esto representa una dificultad aún mayor, quizá no exista algún concepto más ambiguo y más complejo que el de la noción de arte. Entonces, dependiendo del momento histórico y del lugar en que nos encontremos, el arte puede tomar distintas significaciones. Desde posturas que sostienen que el arte es la expresión de algún sentimiento, hasta posturas que toman en cuenta únicamente la técnica del autor, o aquellas que hablan sobre la experiencia estética que se experimenta al conocer la obra; así como también existen las posturas que no toman en cuenta nada de ello.

Para el historiador del arte Jacques Thuiller, nada de ello importa. Señala que ni siquiera existe semántica alguna con la que sea posible relacionar el arte. En todo caso, si, a fin de encontrarse en posibilidad de conceptualizar dicho término, sería necesario llevar a cabo un análisis técnico del término "Arte". Se remonta a la palabra Ars que en latín significa habilidad o talento, sin embargo, lo anterior resulta igualmente ambiguo (Thuiller, 2014). Cicerón utiliza este término a efecto de denotar cierto estatus que ostente alguna persona. Lo dota de cierto carácter creativo al utilizarlo como ars rhetorica, es decir, como arte de la oratoria (Gombrich, 2016). No obstante, resulta difícil atribuir al concepto de arte un significado determinado.

Por otra parte, el magnífico autor León Tolstoi hace que se piense en las formas de las artes, pero nunca en el arte en sí. En este sentido, si preguntáramos por el arte, desde el hombre vulgar, hasta el experto contestaría: ¡Brava pregunta, el arte es pues, la arquitectura, la escultura, la pintura, la música y la poesía! (Tolstoi, 2012). Sin embargo, pareciera que solo se entiende al arte en tanto que concepto y solo se puede entender más bien al producto de la creación artística. 
Adicionalmente, en un esfuerzo por entender aquello a lo que se denomina como arte, el ruso señala que quizá pueda pensarse como una actividad que produce la belleza, lo cual resulta todavía más problemático (Tolstoi, 2012). Por ello, el británico Ernst Gombrich señala que no existe el arte, al menos no "el Arte" con mayúscula: lo que existe son los artistas. Todas aquellas personas que van desde quienes cogían tierra y dibujaban formas de animales en las paredes de una cueva, hasta quienes trazan carteles en las paredes del metro (Gombrich, 2016).

Lo anterior resulta igualmente complejo. Quizá tampoco exista una definición de quién es un artista y, según el autor, tampoco hay nada malo en llamar artista a cualquier persona que realice alguna actividad. Sin embargo, hay que tomar en cuenta que ello puede significar cosas distintas en lugares y temporalidades distintas (Gombrich, 2016). Ahora bien, si se considera al arte como la producción de lo bello, cualquier peluquero o cualquier obrero podría ser considerado como artista.

Ello suponiendo, en primer lugar, que en efecto se pueda definir así al arte; qué ocurriría con una obra de teatro que no sea bella, o con una canción que no sea agradable al oído. Se puede pensar únicamente suponiendo que existe un concepto universal de lo bello pero no es posible. Esto lleva a concluir que definir al artista resulta igualmente imposible que definir lo que es el arte.

\section{La experiencia de los sentidos}

Por otra parte, una obra de arte puede ser realizada por una o muchas personas. Aquellos que buscan expresar algún sentimiento religioso, alguna militancia política, algún miedo o desesperación. Incluso, puede existir una obra de arte que perdure físicamente por milenios o que se termine en segundos. Por ejemplo, teóricos como Kralik señalan que existe un pentáculo que dota al arte como tal, se funda en la experiencia de los sentidos, por lo que puntualiza que al arte lo definen el gusto, el tacto, el oído, el olfato y, por supuesto, la vista (Tolstoi, 2012).

Entonces, ¿qué es aquello que ha impedido entender a la gastronomía como un arte? Sin duda, al disfrutar algún platillo (comerlo o prepararlo) se está experimentando una esteticidad que involucra a todos nuestros sentidos. Por 
consiguiente, preponderantemente se ha enfatizado en el sentido del gusto al describir la experiencia estética relacionada con algún alimento. Sin embargo, en la mayoría de las veces el primer sentido que se estimula es la vista al identificar algo como alimento, ya sea desde el más exquisito platillo preparado muy cuidadosamente, al ver un pescado recién sacado del agua, o una colorida fruta colgando de un árbol.

De la misma manera el olfato, pues ya desde lejos se puede percibir un aroma sin haber visto antes el alimento. No importa que haya sido expulsado por el alimento al elevar su temperatura o simplemente por su olor natural, incluso asociado al sabor, es decir, se puede interpretar un olor como dulce, como picante o como ácido.

Una vez comenzada la experiencia culinaria, el espectador (el comensal) inmediatamente activa su sentido del tacto; generalmente, la textura de la comida se siente en la boca y en otras ocasiones, otras partes del cuerpo. Esto es, si es crujiente o suave, si es jugosa o seca. Aunque habitualmente no se menciona el sentido del oído, no se puede perder de vista los nombres otorgados a experiencias del tacto como lo crujiente, haciendo una clara alusión al sonido que provoca al comerlo.

Lo anterior, sin mencionar la importancia del sonido que emanan los alimentos tras su preparación. Por ejemplo, imagine la experiencia de una carne asada; empieza al escuchar a los tejidos cambiando por el calor, o algún postre sacado del aceite caliente y que aún se puede escuchar su crujir al freírse. Ahora, aunque se deje de lado el carácter estético del arte, no cabe duda la cantidad de sentimientos e ideas transmitidos por medio de la comida, pues además del fuerte contenido nacionalista que existe en cada platillo, sin importar incluso la militancia política que representan en la mayoría de los casos, el cocinero, chef, genio, artista o cualquiera que sea el término que se desee utilizar, emplea toda clase de técnicas para transmitir al comensal sus sentimientos o ideas.

El mismo Sartre en su obra El ser y la nada describe la carga psíquica de los alimentos y pone como ejemplo lo viscoso. Señala que desde su punto de vista, lo viscoso se asocia siempre con lo desagradable, lo que resulta un claro ejemplo de la forma en la que intervienen nuestras pasiones y nuestros deseos en la forma en que se comprende cualquier cosa. Desde la abstracción que realizamos de una propiedad como lo viscoso, hasta la forma en que imaginamos un pastel rosado. 
El autor describe el sabor y la textura que se imagina al pensar en un pastel rosado. No obstante, resulta claro que al señalar lo anterior evidencia la parte pasional de cualquier persona al entender cualquier concepto. Al imaginarme un pastel rosado pienso, por ejemplo, en el sabor y el olor del colorante, mientras que otra persona puede pensar en fresas u otros frutos (Sartre, 2002).

Por mencionar algunos otros ejemplos, la cocinera afroamericana Mashama Bailey, quien creció en el sur de los Estados Unidos, emplea ingredientes de esa región para elaborar platillos comidos por los esclavos en el estado de Georgia. Actualmente ha ganado fama pues gente de todo su país viaja a ese estado para probar sus platillos sin darse cuenta de que ello constituye una clara reivindicación de la cultura y de las costumbres de la población afroamericana de esa región.

Más claro resulta aún el caso del repostero español Jordi Roca. Ha sido considerado por varias autoridades en el campo de la gastronomía como el mejor repostero del mundo. Uno de sus platillos más conocidos es un pastel sabor a olor a tierra. Este postre ha causado mucho revuelo pues la gente que lo prueba queda enamorada de él. El famoso repostero señaló que la clave del éxito de este postre es que en la región de España en la que vive y creció (Girona) no hay una persona a la que el olor a tierra mojada no le recuerde a su infancia. Entonces, quien prueba este pastel libera en su mente toda la carga psíquica que representan sus recuerdos, ilusiones y deseos de su niñez.

De la temporalidad ni hablar pues, al igual que cualquier arte performativo, o cualquier "performance" que Yoko Ono realice en Central Park; el espectador experimenta ciertas sensaciones y pensamientos por algunos momentos. Después estos se esfuman, quedando únicamente en su mente los recuerdos y reflexiones de su experiencia; tal y como sucede con la música, así como con el teatro (cuyas obras son efímeras), cualquier platillo, ya sea comido o no, su punto de perfeccionamiento no dura más que un instante.

Aunque pareciera que las recetas perpetúen en el tiempo alguna obra gastronómica, únicamente sucede lo mismo que sucede con cualquier partitura y la música que pudo haber deleitado a los antepasados, o al igual que con las obras de teatro escritas hace años y que son representadas en cualquier momento. Así, resulta claro que cualquiera que sea el criterio de determinar si algo puede ser considerado como arte o no, es sin duda aplicable a la gastronomía, lo cual hace pensar de nueva cuenta, cuál fue aquel criterio que descalificó históricamente a la gastronomía en el campo de las artes. 
La respuesta quizá pueda encontrarse no en la gastronomía misma ni en el intento de una conceptualización del arte, mejor en los procesos históricos mediante los cuales se ha definido y redefinido al arte. Por consiguiente, se debe considerar la importancia que ha tenido el pensamiento platónico en la conformación de nuestra cultura occidental. En primer lugar, se debe entender cómo Platón concibe la belleza, y es que en algunos de sus diálogos como en "el Fedro" o "el Banquete", hace referencia a esta en varias ocasiones. Sin embargo, en ningún momento lleva a cabo ninguna reflexión en torno a la belleza como arte, más bien habla de la belleza que alguna persona posea.

Sin embargo, pareciera que derivado de lo reflexionado por Platón en sus diálogos, se entiende que al poder amar algo solo por el hecho de ser partícipe de lo bello; en este sentido, la belleza es pensada como una idea y no como algún tipo de accidente (Thuiller, 2014). También vale la pena recordar que en el sistema platónico son las ideas las que deben de ser privilegiadas desde un punto de vista metafísico, dejando lo corpóreo o lo finito de lado por completo, como si se tratara únicamente de las sombras de las cosas. Así, cuando en la discusión llevada a cabo en el Fedon se hace referencia a la música y se dice que "en la lira afinada la armonía es cosa invisible, incorpórea, enteramente bella y divina" (Thuiller, 2014). Deja claro la forma en que la belleza (o lo bello) para Platón no puede ser algo físico, sino algo completamente incorpóreo y eterno.

En este orden de ideas, vale la pena mencionar la forma en que el mismo Platón, en su libro X de La república desprecia por completo a los poetas y a todas las personas dadas a la imitación. Únicamente se dedican a representar la apariencia de las cosas y no lo que en verdad son (Thuiller, 2014). En ese sentido, existe una idea de la manera en que claramente el sistema de pensamiento platónico desdeña lo corpóreo. El "elogiar" la reproducción de un arte (como en este caso la música) proveniente de la lira dando con calificativos como incorpórea, invisible y divina. Esto da a entender que las artes o técnicas con resultados corpóreos deben de quedar en un nivel menos privilegiado. El mismo Eugenio Verón señala:

No hay ciencia que, como la estética, se haya prestado a las lucubraciones de los metafísicos. Desde Platón hasta las doctrinas actuales de nuestros días, se ha hecho del arte una informe amalgama de misterios trascendentales y de teorías llevadas a la quinta esencia, que hallan su expresión suprema en la concepción absoluta de lo bello ideal, prototipo inmutable y divino de las cosas reales. (Tolstoi, 2012) 
Por otra parte resulta necesario mencionar la resonancia tan considerable que tuvo lo anteriormente descrito para el pensamiento medieval. En primer lugar, se conoce la importancia que el pensamiento platónico tuvo para la formulación de ciertos conceptos importantes en el cristianismo, por lo que la idea de lo bello y del arte no fue la excepción. Adicionalmente, no se puede perder de vista el carácter teológico del arte que nació en la Edad Media, claramente influenciado por ideas platónicas y neoplatónicas. Como se observa, ideas como la de lo bello (planteadas por autores como Plotino, Proclo o el abad Suger de Saint Denis), incluso cuando su concepto de belleza no es un concepto teológico, como sucede en el caso de los primeros dos (2), su influencia neoplatónica ayudó a configurar una noción del arte completamente teológica.

En vista de lo anterior, no se puede perder de vista la forma en que Plotino identifica la belleza con el "uno"; el primer principio que es origen y causa de todos los seres, lo que los analistas cristianos posteriores identificaron fácilmente como Dios. De esta manera, para Plotino el "uno" es a la vez el bien y la belleza absolutos; en cambio, la belleza material consiste en la participación de la belleza del "uno". Así, los seres corpóreos serán más bellos en tanto que más se aproximen a la fuente de belleza primera. Por otro lado Proclo, tomando como base mucho de lo sostenido por Plotino, analiza el carácter de la belleza divina. La identifica con la idea del Bien y sostiene que esta belleza divina es la causa de la belleza que poseen los seres materiales, dándoles el mismo significado a estos conceptos que les dio Platón en el Fedro (Tomasini, s.f.).

Por último, vale la pena tomar en cuenta lo señalado por el abad francés. Ya en el siglo XII, sostiene que el alma es incapaz de alcanzar la verdad sin ayuda de representaciones visuales. Además, que el espíritu es conducido a la contemplación de lo inmaterial por medio de las imágenes materiales (Tomasini, s.f.). En primer lugar, ello brinda una idea de la importancia que tuvieron las ideas platónicas, luego, la teología para la configuración del concepto de lo bello para occidente (por lo tanto del arte). No puede caber duda del carácter teológico que estas ideas dotaron al arte no solo en la Edad Media, sino para toda la historia.

Lo anterior es así, pues si bien a la fecha el pensamiento artístico puede haberse secularizado, derivado de distintos procesos dialecticos materialistas y sociales; su carácter teológico siempre va a ser producto los procesos de historicidad que lo constituyen, por lo que difícilmente se puede pensar en un arte completamente secular. Ahora bien, al hablar de la gastronomía resulta un saber contrario al ideal 
artístico neoplatónico y medieval. Además de que la gastronomía constituye a todas luces una producción material (lo que en primer lugar se aleja del ideal platónico), contrario a la pintura o a la arquitectura que en la Edad Media sirvieron para acercar al alma la divinidad; tanto la producción gastronómica, como su contemplación (la experiencia estetizada de alimentarse) constituyen en sí mismas un pecado mortal.

\section{La contemplación gastronómica}

En este sentido, al tratarse de un arte, o mejor de una actividad, cuyo fin último es el de brindar placer a los sentidos; no es de extrañarse que el mismo Tomás de Aquino clasificó a la gastronomía (o como él le llama: la glotonería) como uno de los pecados capitales en el cristianismo. No solo es eso, además en su Summa Teológica incluso menciona que es uno de los pecados más graves, no solo se limita a alejar al hombre de la virtud en términos teológicos; también lo induce a otros pecados como la soberbia y la lujuria, por lo que castigos como el diluvio o la destrucción de Sodoma fueron derivados de la Gula (De Aquino, 1998).

Es decir, para Tomás de Aquino tanto la producción, como la contemplación gastronómica, representan un placer tal que incita al individuo a perder cualquier clase de comportamiento moderado. Por consiguiente, no solo lo aleja de la virtud (por lo tanto de la contemplación de lo divino), incluso lo induce a cometer otro tipo de pecados mortales. Entonces, ¿cómo podemos considerar algo que no solo se aleja de la contemplación de lo divino que constituye un pecado mortal y que gracias a este fueron originados los dos (2) castigos más brutales descritos en la Biblia como una forma de arte?

Quizá visto desde un punto de vista teológico, la gastronomía debería ser considerada como el anti-arte por antonomasia. Emplean técnicas, sentimientos y, como se ha descrito, una experiencia estética que involucra todos los sentidos, pero que no solo aleja de lo divino, sino que incluso conduce al diluvio. Es decir, la gastronomía (al igual que la poesía o la pintura) reúne los criterios empleados para conceptualizar al arte. Sin embargo, a diferencia de estos, en lugar de acercarse a la divinidad aleja de ella y conduce al pecado. 
Según lo anterior, resulta claro por qué la gastronomía no ha sido inscrita bajo el rubro de las bellas artes; incluso en la actualidad, cuando se habla de arte, sin importar los procesos secularizantes que han tenido lugar en esta modernidad tardía. Cuando se habla de gastronomía se piensa en ciencia, en economía y en un sin número de saberes, pero no se piensa en arte; así como cuando se habla de arte, no se menciona a la técnica gastronómica.

La prueba de ello es lo que pareciera un desdén a las vanguardias como el Eat Art, que intenta introducir elementos gastronómicos a los happenings llevados a cabo en los museos. Aun suponiendo que dichas corrientes gozaran de reconocimiento pleno por las autoridades en la materia del arte, lo único que tratan es introducir ciertos elementos que complementen a la experiencia del espectador. Por lo tanto, tampoco se habla de la técnica gastronómica en sí, o de lo que esta representa.

Entonces, desde la última cena de Leonardo Da Vinci, hasta las naturalezas muertas de Cézanne, se ha pensado a la gastronomía dentro del arte. Sin embargo, esto pareciera que se trata únicamente de un esfuerzo que podría sonar incluso hasta elitista por parte de los artistas. Lo anterior, por admirar a la comida desde las alturas, como si se tratara de alguna necesidad básica del ser humano y no de una técnica elevada como lo es el arte. Por último, vale la pena mencionar la polémica anécdota ocurrida en el año 2007, cuando el curador de arte Roger Buergel extendió una invitación al famoso chef español Ferran Adriá para participar en la reconocida Documenta de Kessel.

Adriá es reconocido por su peculiar estilo de cocina, ha introducido elementos y técnicas que han revolucionado por completo la cocina contemporánea. En estos términos, el curador consideró que vendría bien presentar una técnica y un diseño gastronómico que rompa con lo convencional en dicha exposición. Según algunos teóricos, esa forma de cocina se encuentra más cerca de las vanguardias artísticas experimentales que de la misma gastronomía.

Cabe mencionar que, de nuevo, se vuelve a la misma disyuntiva mencionada anteriormente. Una vez más, pareciera que el artista se encuentra reconociendo desde las alturas al cocinero y lo invita a participar como su igual. Sin embargo, solo dentro de las disciplinas artísticas tradicionales, nunca desde el punto de vista estricto de la gastronomía. 
Sea como sea, el famoso chef contestó lo siguiente: "Allí voy en representación de todos, porque el debate va a ser si la alta cocina puede codearse con otras 'altas' artes. Sé que sólo soy cocinero y voy ahí desde la humildad". Asimismo, señala:

No soy Picasso ni lo pretendo; comprendo que haya gente que se moleste. Sé que es duro que inviten a un cocinero. Pero, ¿qué es arte? No lo sé. Si a esto quieren llamarlo arte, muy bien. Si no, también; eso no depende de mí. (Cuculiansky, 2007)

\section{Conclusiones}

Así, resulta sumamente complicado concluir si se puede incluir a la gastronomía bajo el rubro de las bellas artes o no. No cabe duda de que la gastronomía no se trata solo de satisfacer una de las necesidades más básicas del ser humano; al igual que cualquiera de las bellas artes, involucra altísimas técnicas tanto creativas como de elaboración y de ingenio, ya sea en el más fino comedor o en el puesto de comida callejera más común. Como se ha evidenciado, es quizá una de las experiencias estéticas más intensas que cualquier ser humano pudiera experimentar; de una manera muy enfática involucra a todos los sentidos.

Ni hablar de la expresión que representa el cocinero al preparar sus platillos. No solo es un ejemplo de añoranza a la tierra, también puede expresar su deseo revolucionario llegando al grado, incluso, de sugerir por medio de un platillo la necesidad de la militancia armada. Sin embargo, como se ha evidenciado resulta sumamente interesante determinar si es posible considerar a la gastronomía como una de las artes más sublimes, o más bien, como el anti-arte por antonomasia. Ya que produce la misma experiencia sublime que puede llegar a producir la más bella pintura o la más dulce melodía, no obstante, en lugar de "hacernos" participes de la divinidad, hace participes del pecado.

Sea como sea, no cabe duda de que la gastronomía ha acompañado al ser humano desde su primera prehistoria. Además, su carácter estético es tal que ha tomado un importante rol en la configuración de cualquier tipo de relación social y política, ya sea que los participantes de estas relaciones estén conscientes de ello o no. Así, ya sea que se desee considerar a la gastronomía como arte, o como el anti-arte, lo único que se puede afirmar con toda certeza es que la gastronomía siempre ha causado el experimento de un placer tal, que nos hace temer provocar la venganza de Dios. 


\section{Referencias}

Cuculiansky, S. (3 de Junio de 2007). Extra/Cocina y vinos ¿Hay arte en la cocina? La Nación. Recuperado de https://www.lanacion.com.ar/lifestyle/ hay-arte-en-la-cocina-nid915344

De Aquino, T. (1998). La gula En Summa Theologica II, Ilae, c.148. Madrid: BAC.

Flandrin, J. (1987). Historia de la Alimentación: Por una ampliación de perspectivas. Barcelona: Manuscrits: revista d'història moderna.

Gombrich, E. (2016). La historia del arte. Phaidon.

Harari, Y. (2014). Sapiens, una breve historia de la humanidad. Milenio. Recuperado de: https://www.milenio.com/opinion/liebano-saenz/ lecturas-esenciales/sapiens-historia-humanidad-libro-yuval-harari

Hipócrates. (s.f.). Sobre la dieta, Libro I.

Lévi-Strauss, C. (2012). Mitológicas III: Breve tratado de etnología culinaria. Madrid: Siglo XXI Editores.

Sartre, J. P. (2002). De la cualidad como reveladora del ser En El ser y la nada. Buenos Aires : Losada.

Savarin, J. A. (2016). Fisiología del gusto. España: Evergreen.

Thuiller, J. (2014). Teoría general de la historia del arte. México: FCE.

Tolstoi, L. (2012). ¿Qué es el arte? Valladolid: MAXTOR.

Tomasini, C. (s.f.). El simbolismo de la luz en el Arte Medieval. Academia. Recuperado de https://www.academia.edu/12981465/El_simbolismo_ de_la_luz_en_el_Arte_Medieval 